\title{
Magnetic Properties and Microstructures of Rapidly Solidified FePd Alloy Ribbons
}

\author{
Yoichi Kishi $^{1,}$, ${ }^{\text {, Zenjiro Yajima }}{ }^{1}$ \\ Teiko Okazaki², Yasubumi Furuya² \\ and \\ Manfred Wuttig ${ }^{3}$ \\ ${ }^{1}$ Research Laboratory for Integrated Systems, AMS R and D Center \\ Kanazawa Institute of Technology, 3-1 Yatsukaho, Hakusan, Ishikawa 924-0838, Japan \\ ${ }^{2}$ Faculty of Science and Technology, Hirosaki University, Hirosaki 036-8561, Japan \\ ${ }^{3}$ Department of Materials Science, University of Maryland, College Park, MD 20742, USA \\ akishi@neptune.kanazawa-it.ac.jp
}

Keywords: ferromagnetic shape memory alloy, magnetostriction, martensitic transformation, transmission electron microscope

\begin{abstract}
It is well known that FePd alloys are effective as a magneto-thermoelastic actuator material, because they have large magnetostriction and shape memory effect. In order to use the alloys for a micro-actuator, magnetic properties and microstructures have been examined as for rapidly solidified Fe-29.6 at\% Pd alloy ribbons. The ribbons exhibit a large magnetostriction at room temperature and good shape memory effect. Magnetostriction and coercive force of the ribbons markedly depend on the direction of the applied magnetic field. Maximum values of magnetostriction and coercive force are obtained at $\theta=85$ degree ( $\theta$ is the angle between the magnetic field and the ribbon plane). Relief effects corresponding to the formation of FCT martensite variants are observed on the grains. X-ray diffraction profile at room temperature shows that FCT martensitic phase and FCC parent phase coexist in the ribbon. Dense striations are observed in the TEM bright field images of FCT martensite plates. Selected area electron diffraction patterns revealed the striations to be thin twins.
\end{abstract}

\section{Introduction}

Ferromagnetic Shape Memory Alloys (FMSMAs) form a new class of functional materials, because they show ferromagnetic nature plus shape memory effect and pseudoelasticity. Therefore, two types of energy, i. e. magnetic and thermal, can be used to control their shape memory behavior. Large displacement, which results from the re-arrangement of martensite variants induced by applied magnetic field, promise new applications in the area of Micro Electro Mechanical System (MEMS). Recently, it has been reported that single crystals of ferromagnetic $\mathrm{Ni}_{2} \mathrm{MnGa}$ [1], FePd [2, 3] and FePt [4] alloys under a magnetic field cause a strain of a few percent by the re-arrangement of martensite variants [5]. However, these crystals are either expensive or brittle in manufacturing thin plate and/or wire actuators at present. In addition, their martensitic transformation temperatures are often lower than room temperature.

In our previous studies [6, 7], we reported that polycrystalline FePd FMSMA ribbons made by rapidly solidified melt-spinning showed a good ductility, strong crystal anisotropy, giant magnetostriction as well as shape memory effect. In this paper, in order to further examine the anisotropy, magnetic properties and microstructures of FePd ribbons prepared by rapidly solidified melt-spinning are investigated. 


\section{Experimental Procedure}

An ingot of the FePd FMSMA was prepared by arc-melting in argon atmosphere. Rapidly solidified 40 to $60 \mu \mathrm{m}$-thickness and $5 \mathrm{~mm}$-width ribbons of the alloy were obtained from the ingot by an electro-magnetically controlled melt-spinning apparatus. The apparatus and detailed production procedure of the ribbons are described in the previous paper [6, 7]. Chemical composition of the ribbons was determined with an electron probe micro analyzer to be Fe-29.6Pd (at\%). Magnetization loops were determined with a VSM. The angle, $\theta$, between the direction of applied magnetic field and width direction $(w)$ of the ribbon could be adjusted to investigate the effects of texture and/or shape anisotropy. The magnetostriction, $\quad \varepsilon$, was measured by strain-gauge attached on the sample which was set in a furnace between the electromagnets (see Fig. 1). Martensitic transformation temperatures of the ribbons were examined by a differential scanning calorimeter (Rigaku DSC 8230). Surface and cross sectional morphologies were observed by using a differential interference optical microscope (OM) with cooling/heating stage and a scanning electron microscope with a field emission electron gun (Hitachi S-4200, SEM). Internal microstructures of martensites were examined by using a transmission electron microscope (JEOL-2000FX, TEM) operated at $200 \mathrm{kV}$. Specimens for the TEM observations were mechanicallyand ion-polished, and all the TEM observations were done at room temperature.

\section{Results and Discussion}

The magnetization loop of the ribbon determined at $\theta=0$ degree ( $\mathrm{H} / / w)$ and $\mathrm{H} / / \mathrm{l}$ show a rapid increase of the magnetization

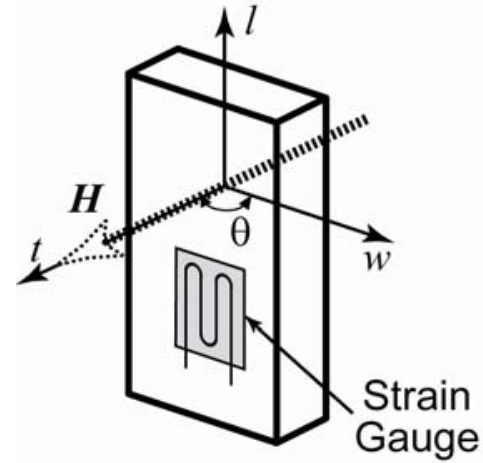

\section{$l$ : Longitudinal Direction \\ $t$ : Thickness Direction \\ $w$ : Width Direction}

Fig. 1: Schematic illustration of mesurement method for magnetic properties.

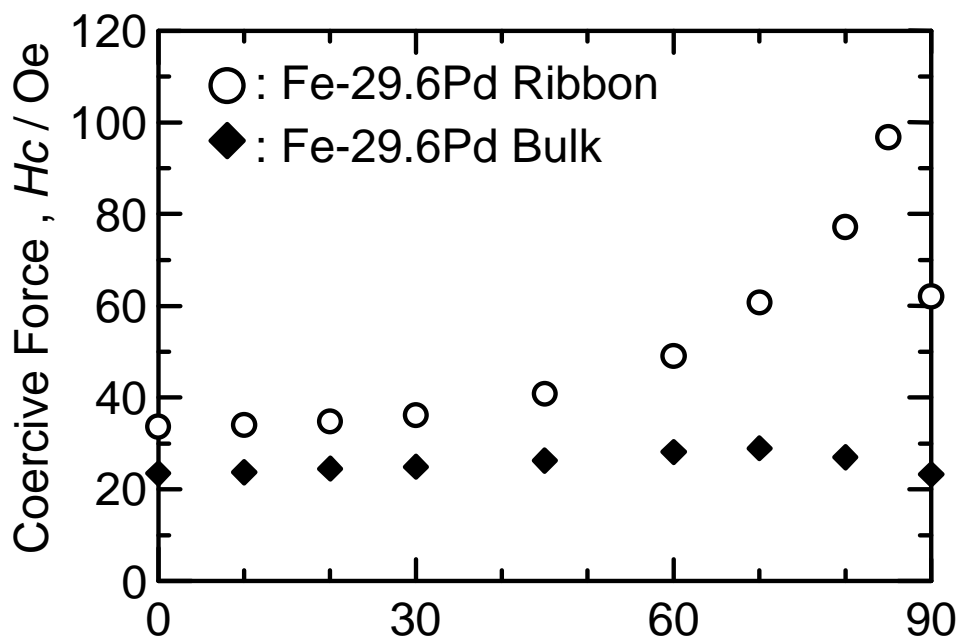

Direction of Applied Magnetic Field , $\theta$ / degree

Fig. 2: Relation between coercive force and applied magnetic field direction for ribbon and bulk samples. All data were obtained at room temperature.

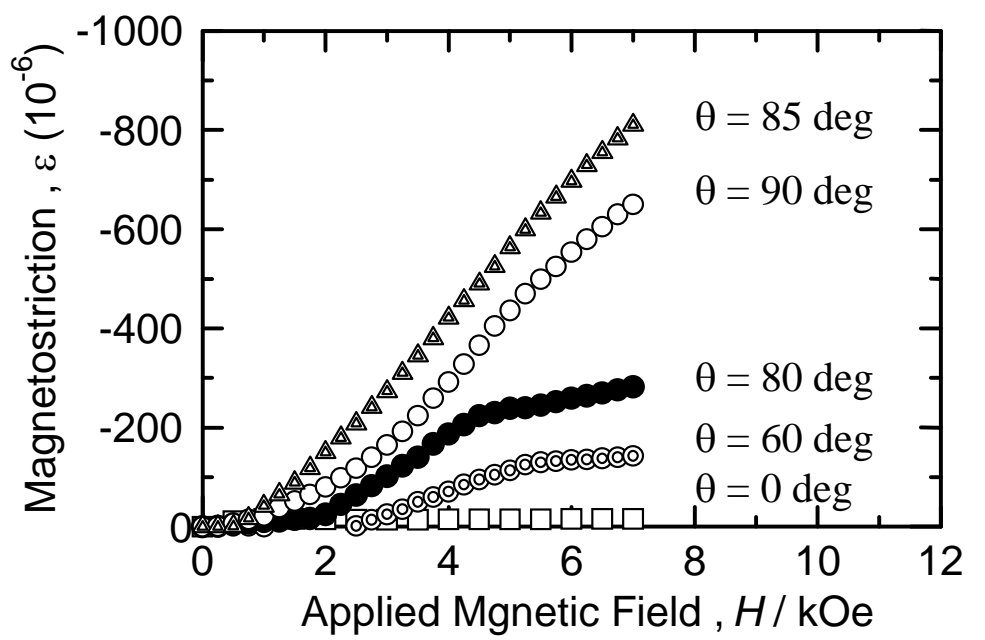

Fig.3: Applied magnetic field direction dependence of magnetostriction measured at room temperature for a ribbon obtained at room temperature. 
saturating at about $5 \mathrm{kOe}$. The coercive force of the ribbon is less than 35 Oe for this angle. For $\theta=90$ degree, however, the magnetization increases linearly up to $5 \mathrm{kOe}$ because of the large demagnetization field. The coercive force is now about 2 times larger than that for $\theta=0$ degree. Contrarily, the direction of applied magnetic field hardly affects the loop of the bulk because of its near isotropic dimensions. Figure 2 shows the anisotropy of the coercive force in ribbon and bulk samples. The coercive force of the ribbon samples markedly depends on the applied magnetic field direction. The maximum coercive force is obtained at $\theta=85$ degree. A similar dependency is observed in relationship between magnetostriction and applied magnetic field direction. Figure 3 shows the dependence of magnetostriction, $\varepsilon$, on applied magnetic field, $\mathrm{H}$, of a ribbon sample. The value of $\varepsilon$ also depends on applied magnetic field direction. The maximum value is $-8.3 \times 10^{-4}$ at $\theta=85$ degree. It thus appears that this phenomenon is characteristic of the rapidly solidified ribbons.

Figure 4 shows Bird-view SEM images of a

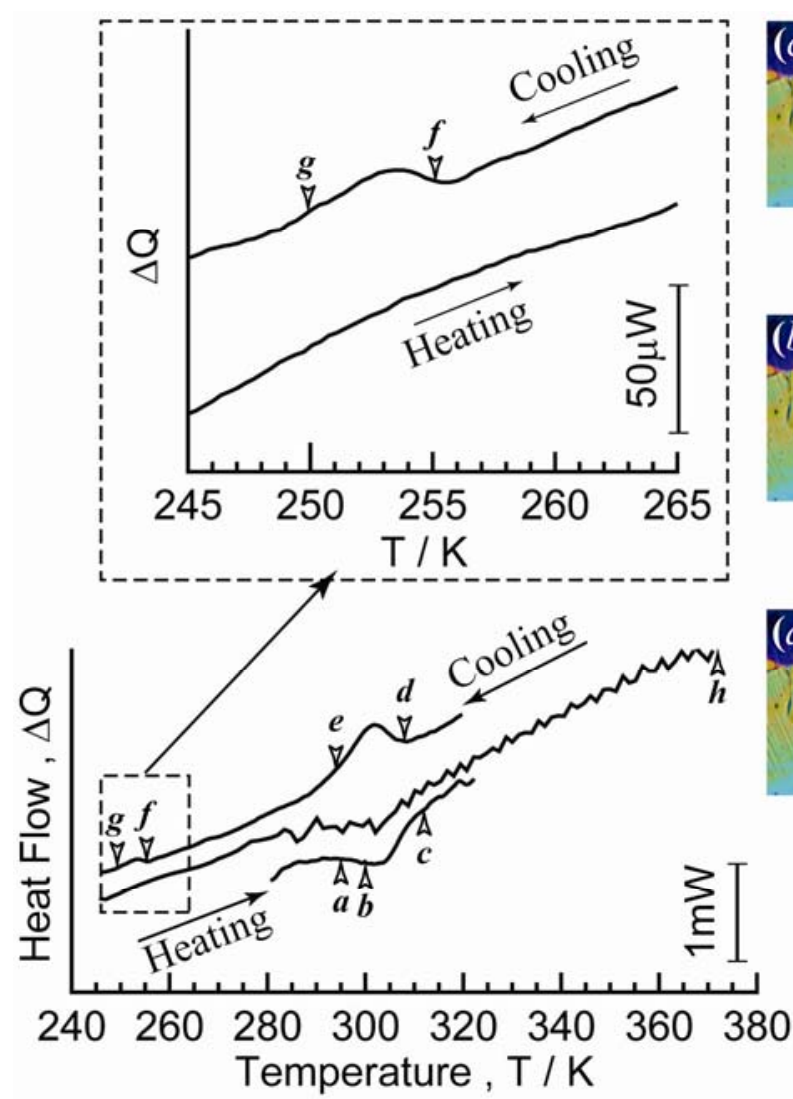

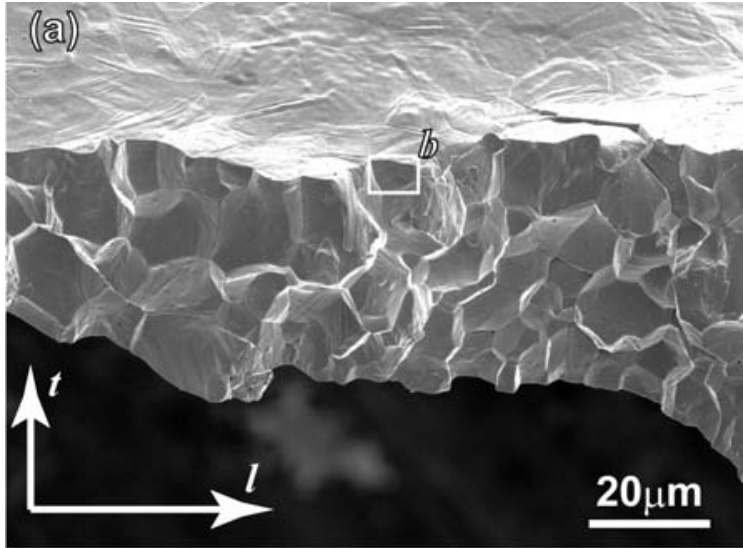

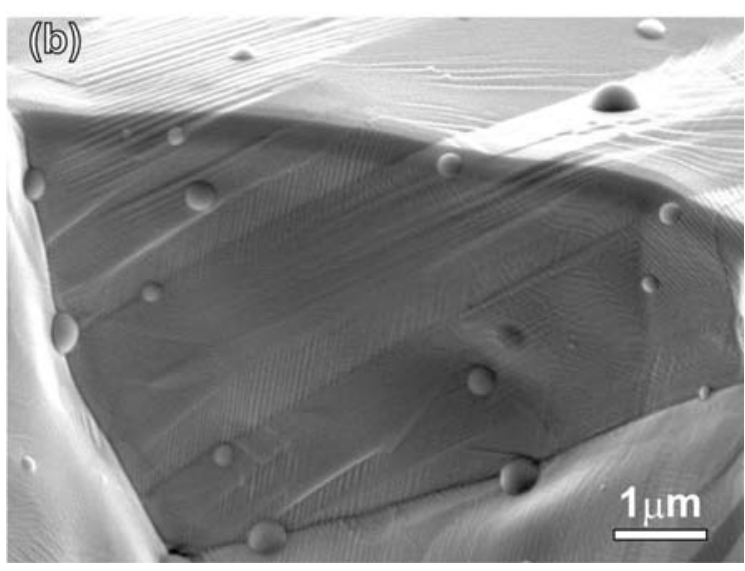

Fig. 4: Bird view SEM images of a ribbon sample. (a) Low magnification image, (b) Enlargement of flamed are b in (a).

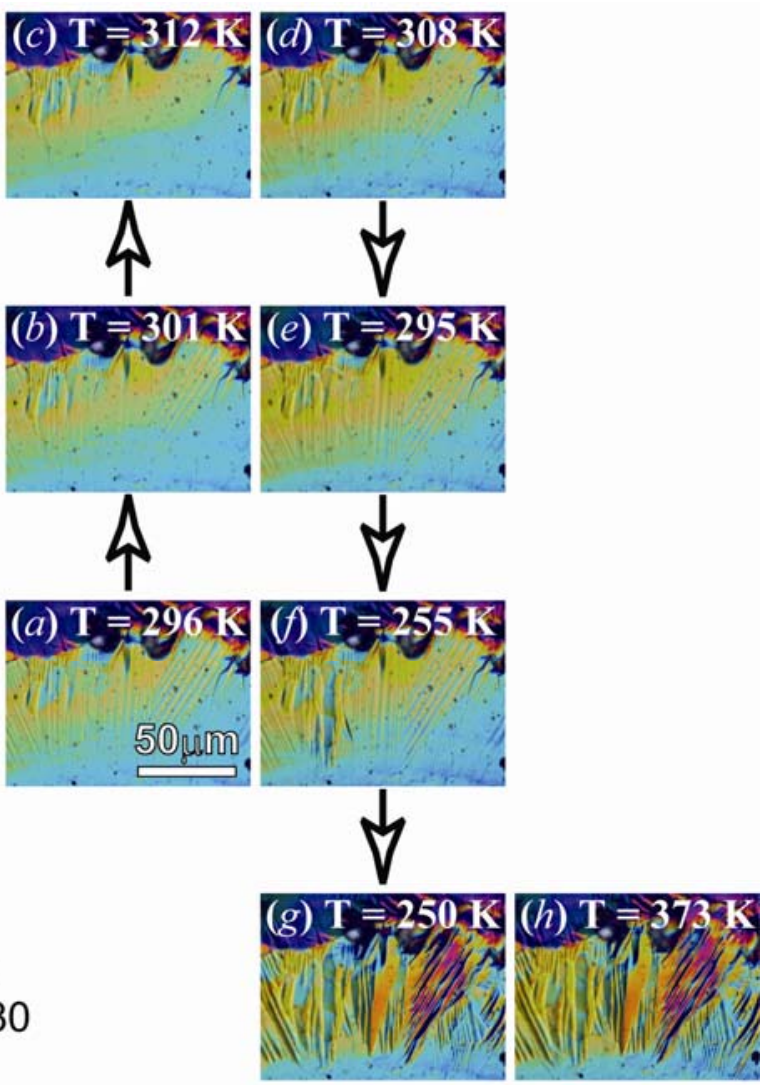

Fig. 5: DSC curves and corresponding surface relief observed during the thermal cycling of a ribbon sample. 
ribbon sample. Grains, whose diameter is about $20 \mu \mathrm{m}$, were observed in the cross section. Second phase particles such as a droplet, which are $\mathrm{Fe}_{\mathrm{X}} \mathrm{Pd}_{1-\mathrm{X}}$ compound, were observed on the surface of the crystal grains (see in Fig. 4 (b)). Relief effects corresponding to the formation of FCT martensite variants were observed on the surface of the crystal grains. Length of the relief on the $w-l$ section appears sometimes to be larger than the grain diameter, because the grain boundaries of the rapidly solidified ribbons consist of low-angle ones.

Heat flow, $\Delta Q$, versus temperature, $T$, diagram for an as-spun ribbon are shown in Figure 5 . An endothermic peak and an exothermic peak are observed on the heating ( $a \rightarrow b \rightarrow c$ in Fig. 5) and cooling ( $d \rightarrow e$ in Fig. 5 ) process, respectively. These peaks exhibit FCT (martensitic phase) $\leftrightarrow$ FCC (parent phase) transformation. The differential interference OM images are also shown in Figure 5, corresponding to wedge marks on the heating and cooling DSC curves. The martensitic domain boundaries are nearly straight and the growth behavior is thermoelastic, both being typical for shape memory alloys, and similar morphology and growth behavior have been observed for the martensites in CoNiGa bulk and ribbon FMSMAs, as already reported in previous papers [8-11]. When the cooling process is further continued, an exothermic peak and well-developed surface relief, which are corresponding to BCT martensite variants, can be observed. The BCT martensitie variants remains

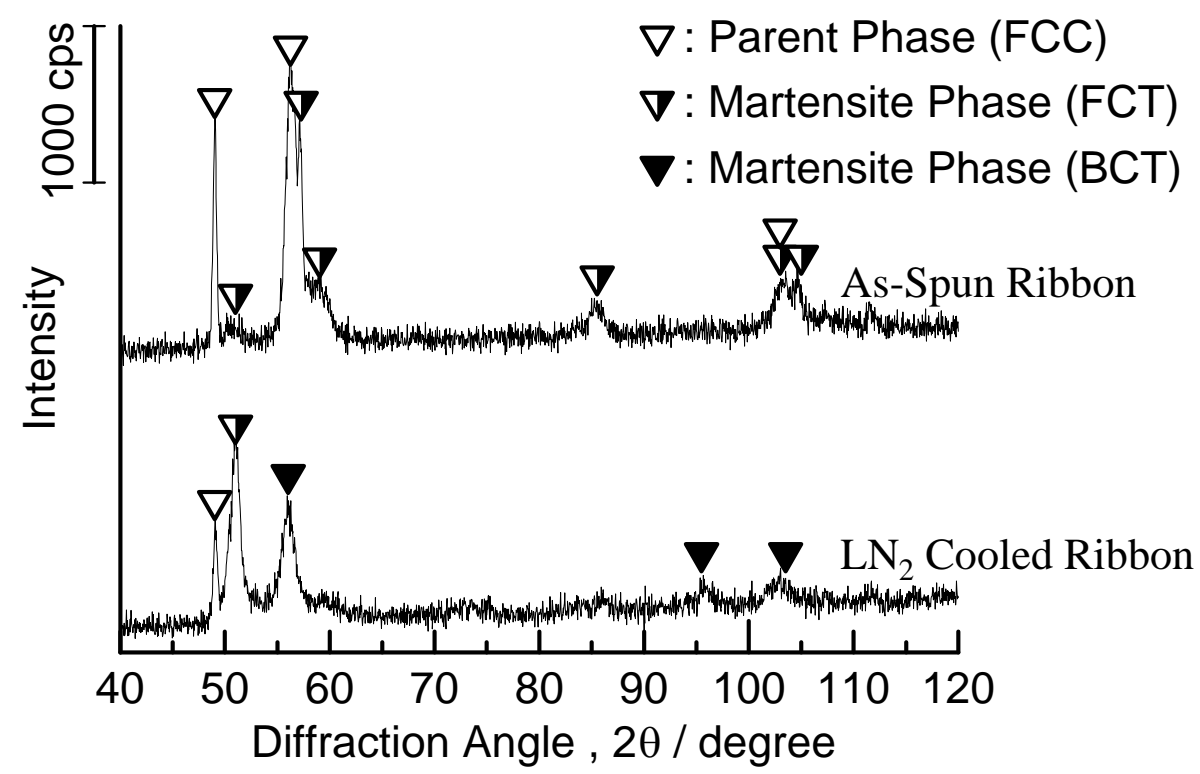

Fig. 6: X-ray diffraction profiles with Co-K $\alpha$ radiation of as-spun and LN2 cooled ribbons obtained at room temperature.

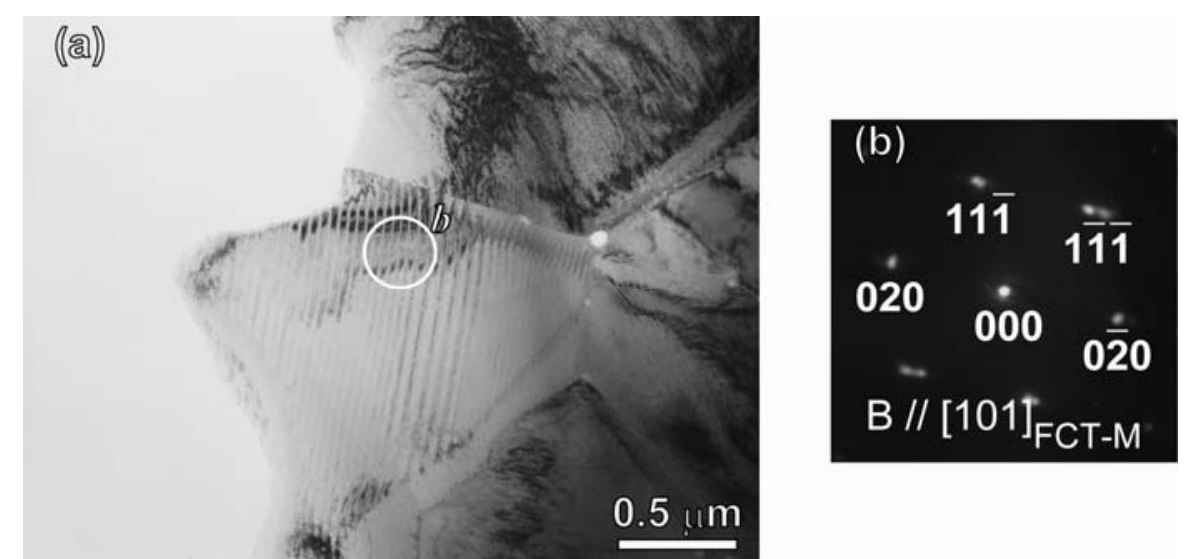

Fig. 7: TEM images of as-spun ribbon sample. (a) Bright field image, (b) SAED patterns taken of circled area $b$ in (a). 
even after the ribbon is heated up to $373 \mathrm{~K}$, as shown in Fig. 5 (h). Corresponding to this heating process, a noise signal overlapped with the DSC curve. Its significance is unknown at this time.

$\mathrm{X}$-ray diffraction profiles with Co-K $\alpha$ radiation were mesured at room temperature. Figure 6 shows X-ray diffraction profiles of as-spun and $\mathrm{LN}_{2}$ cooled ribbons. The as-spun ribbon was composed of FCC parent and FCT martensitic phases. During LN2 cooling process, a part of FCC parent and/or FCT martensitic phases transformed to BCT martensitic phase. FCC parent phase and FCT martensitic phase were retained in the ribbon sample after LN2 cooling process. Therefore, the LN2 cooled ribbon consists of FCC parent, FCT martensitic and BCT martensitic phases.

Figure 7 shows a TEM image of martensites in an as-spun ribbon. This image was obtained with electron beams parallel to the $t$ direction. High-density of striations are observed in the FCT martensites and twin-related two sets of reflections are observed in the SAED patterns taken of the martensites, accompanying by streaks. Mirror plane of the twinned reflections is the $\{220\}$ plane, and the streaks are perpendicular to the striations in the TEM images. These geometrical reflections clearly indicate that the striations are fine $\{220\}$ twins of the martensites, as observed in many other alloys.

\section{Summary}

The magnetostriction and coercive force of the Fe-29.6Pd ribbon strongly depends on the direction of the applied magnetic field. These maximum values are obtained at $\theta=85$ degree. Relief effects corresponding to the formation of FCT martensite variants were observed on the surface of the crystal grains. The martensitic domain boundaries are nearly straight and the growth behavior is thermoelastic, both being typical for shape memory alloys. During LN2 cooling process, a part of FCC parent and/or FCT martensitic phases transformed to BCT martensitic phase. FCC parent phase and FCT martensitic phase were retained in the ribbon sample after LN2 cooling process. Therefore, the LN2 cooled ribbon consists of FCC parent, FCT martensitic and BCT martensitic phases. Typical internal twinned microstructures, whose twinning plane is the $\{220\}$ plane, and associated twinned and streaked SAED patterns are observed in the TEM images of the FCT martensite variants.

\section{Acknowledgment}

The present study was partly supported by "High-Tech Research Center” Project (2001-2005) and “Open Research Center” Project (2006-2010) for Private Universities: matching fund subsidy from MEXT. YK, NS and ZY acknowledge the Grant-in-Aid for Young Scientists (B) (16760575) and Basic Researches (C) (19560710, 19560323 and 18560680) from MEXT.

\section{References}

[1]P. J. Webster, K. R. A. Ziebeck, S. L. Town, M. S. Peak: Philosophical Magazine B, Vol. 49 (1984), p. 295

[2] R. Oshima: Scripta Metallurgica, Vol. 15 (1981), p. 829

[3] R. D. James and M. Wuttig: Philosophical Magazine A, Vol. 77 (1998), p. 1273

[4] C. M. Wayman: Scripta Metallurgica, Vol. 5 (1971) 489.

[5] T. Fukuda, T. Kakeshita and T. Takeuchi: Materia Japan, JIM, Vol. 44 (2005), p. 285

[6] Y. Furuya: Proceedings of $1^{\text {st }}$ Japan-France Intelligent Materials and Structures Seminar (October, Sendai, Japan), Ed. by J. Tani and P. F. Gobin, (1997), p. 113.

[7] Y. Furuya, N. W. Hagood, H. Kimura and T. Watanabe: Materials Transactions, JIM, Vol. 39 (1998), p. 1248.

[8]M. Wuttig, J. Li and C. Craciunescu: Scripta Materialia, Vol. 44 (2001), p. 2393 
[9]C. Craciunescu, Y. Kishi, T. A. Lograsso and M. Wuttig: Scripta Materialia, Vol. 47 (2002), p. 285

[10] Y. Kishi, C. Craciunescu, M. Sato, T. Okazaki, Y. Furuya and M. Wuttig: Journal of Magnetism and Magnetic Materials, Vol. 262 (2003), p. L186

[11]Y. Kishi, Z. Yajima, K. Shimizu, T. Okazaki, Y. Furuya and M. Wuttig: Materials Science and Engineering A, vol. 481-482 (2008), p. 442 